\title{
ESC observations of SN 2005cf
}

\section{Optical spectroscopy and the high-velocity features ${ }^{\star}, \star \star$}

\author{
G. Garavini ${ }^{1,10}$, S. Nobili1 ${ }^{1,10}$, S. Taubenberger ${ }^{5}$, A. Pastorello ${ }^{5,8}$, N. Elias-Rosa ${ }^{2,3,5}$, V. Stanishev ${ }^{1}$, G. Blanc ${ }^{2,9}$, \\ S. Benetti ${ }^{2}$, A. Goobar ${ }^{1}$, P. A. Mazzali ${ }^{5,4}$, S. F. Sanchez ${ }^{7}$, M. Salvo ${ }^{6}$, B. P. Schmidt ${ }^{6}$, and W. Hillebrandt ${ }^{5}$ \\ 1 Department of Physics, Stockholm University, AlbaNova University Center, 10691 Stockholm, Sweden \\ e-mail: gabri@physto.se \\ 2 INAF Osservatorio Astronomico di Padova, Vicolo dell'Osservatorio 5, 35122 Padova, Italy \\ 3 Universidad de La Laguna, Av. Astrofísico Francisco Sánchez s/n, 38206 La Laguna, Tenerife, Spain \\ ${ }^{4}$ INAF Osservatorio Astronomico di Trieste, via Tiepolo 11, 34131 Trieste, Italy \\ Max-Planck-Institut für Astrophysik, Karl-Schwarzschild-Str. 1, 85741 Garching bei München, Germany \\ ${ }^{6}$ Research School of Astronomy and Astrophysics, Australian National University, Cotter Road, Weston Creek, ACT 2611, Australia \\ 7 Calar Alto Observatory Centro Astronómico Hispano Alemán C/ Jesús Durbán Remón, 2-2 04004 Almeria, Spain \\ 8 Astrophysics Research Centre, School of Mathematics and Physics, Queen's University Belfast, BT7 1NN, UK \\ 9 APC, Université Paris 7, 10 rue Alice Domon et Léonie Duquet, 75205 Paris Cedex 13, France \\ 10 LPNHE, CNRS-IN2P3, University of Paris VI \& VII, Paris, France
}

Received 19 December 2006 / Accepted 13 February 2007

\section{ABSTRACT}

\begin{abstract}
The ESC-RTN optical spectroscopy data set for SN $2005 \mathrm{cf}$ is presented and analyzed. The observations range from -11.6 and +77.3 days with respect to $B$-band maximum light. The evolution of the spectral energy distribution of SN $2005 \mathrm{cf}$ is characterized by the presence of high velocity Si II and Ca II features. SYNOW synthetic spectra are used to investigate the ejecta geometry of silicon. Based on the synthetic spectra, the Si II high-velocity feature appears detached at $19500 \mathrm{~km} \mathrm{~s}^{-1}$. We also securely establish the presence of such a feature in SN 1990N, SN 1994D, SN 2002er, and SN 2003du. In a morphological study both the Ca II IR triplet and $\mathrm{H} \& \mathrm{~K}$ absorption lines of SN 2005cf show high-velocity features centered around $24000 \mathrm{~km} \mathrm{~s}^{-1}$. When compared with other type Ia SNe based on the scheme presented in Benetti et al. (2005, ApJ, 623, 1011), SN 2005cf definitely belongs to the LVG group.
\end{abstract}

Key words. stars: supernovae: general - stars: supernovae: individual: SN 2005cf

\section{Introduction}

Type Ia supernovae (type Ia $\mathrm{SNe}$ ) are the best standardizable candles currently known and they have proved extremely useful for cosmological studies. In the past decade type Ia $\mathrm{SNe}$ have been used to compute the energy budget of the Universe and to establish the presence of dark energy (Perlmutter et al. 1998; Garnavich et al. 1998; Schmidt et al. 1998; Riess et al. 1998; Perlmutter et al. 1999; Knop et al. 2003; Tonry et al. 2003; Riess et al. 2004; Astier et al. 2006). The observational evidence that brighter/dimmer supernovae have light-curves declining slower/faster (Phillips 1993) allows their use as cosmology lighthouses. Light-curve decline-rate parameters (for example $\Delta m_{15}$ ) have been extensively used to divide type Ia SNe into sub-groups. Their observed spectroscopical diversity, however, cannot be understood using only one parameter. Multi-parameter representations of this diversity based on the spectral morphology (Benetti et al. 2005) or on spectral

\footnotetext{
* All individual spectra are only available in electronic form at the CDS via anonymous ftp to

cdsarc.u-strasbg.fr $(130.79 .128 .5)$ or via

http://cdsweb.u-strasbg.fr/cgi-bin/qcat?J/A+A/471/527

$\star \star$ Tables 1-4 are only available in electronic form at http://www . aanda.org
}

modeling (Branch et al. 2006, 2005) have been proposed. These allow an overview of supernova parameter space but fail to be exhaustive. Supernova examples, such as SN 1999ac (Garavini et al. 2005) or SN 2002cx (Jha et al. 2006), do not fit in the proposed classifications and require a deeper knowledge of supernova physics to be understood. Careful studies of early SN spectroscopic and polarimetric data have highlighted that the external structure of the supernova ejecta could be more complex than once believed. Three-dimensional effects such as explosion asymmetries and local density inhomogeneity could be at the origin of the non-spherically symmetric distribution of the intermediate mass elements sometimes found when inspecting the data. The discovery of high-velocity absorption features (HVFs) in many of the early supernova spectra recently observed (see for example (Mazzali et al. 2005a, see for example) have pushed the development of more complex fully 3-dimensional numerical simulations of supernova explosions (see for example Röpke et al. 2006).

In this work we present and analyze the optical spectroscopy data set of SN 2005cf acquired by the European Supernova Collaboration part of the type Ia SNe Research Training Network (ESC-RTN $\left.{ }^{1}\right)$. The almost daily spectroscopic

\footnotetext{
1 http://www . mpa-garching.mpg.de/ rtn/
} 


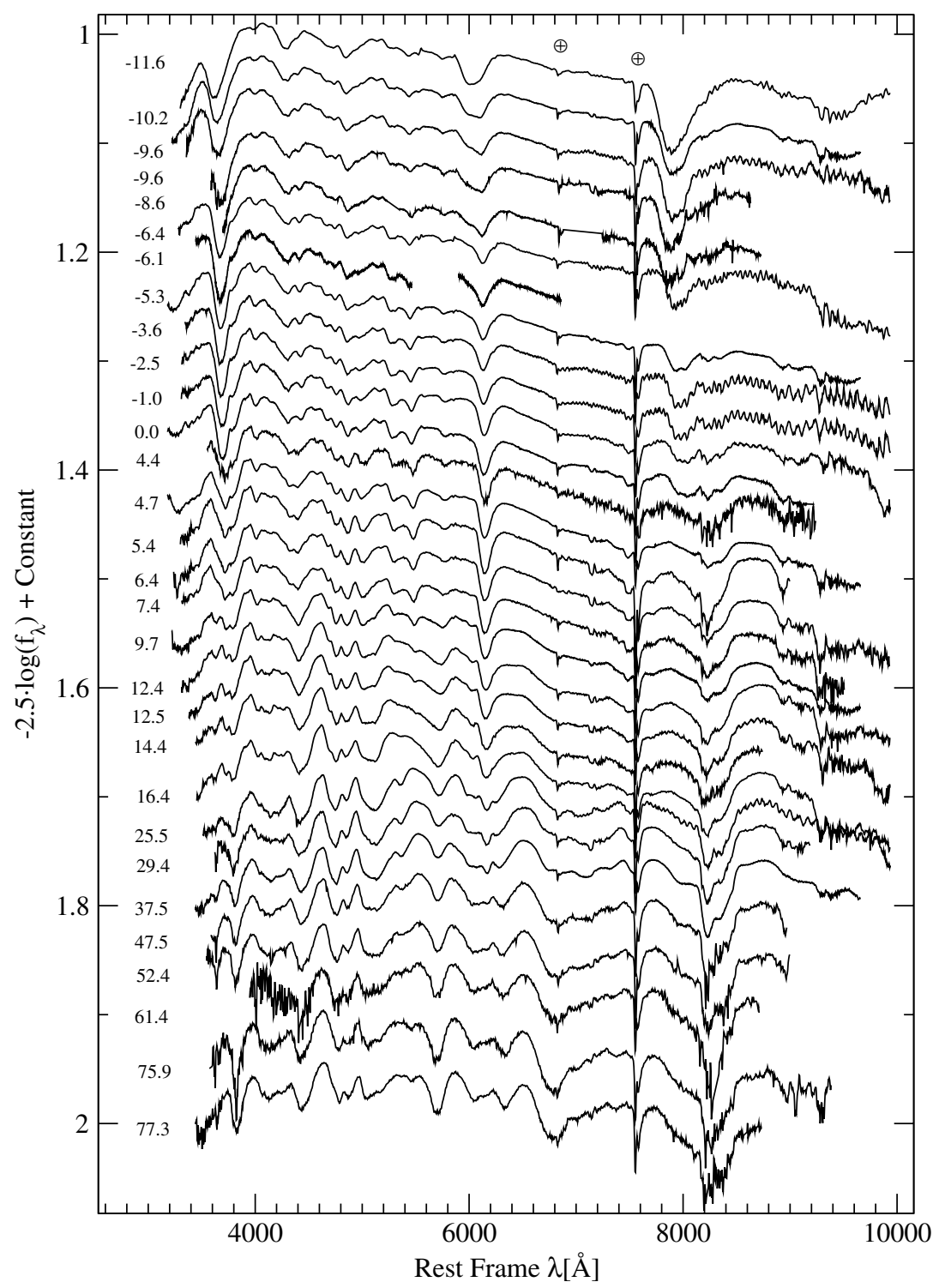

Fig. 1. SN 2005cf spectral time sequence. The flux spectra are shown in rest frame. The epochs with respect to $B$-band maximum light are reported on the left end side of each spectrum. The $\oplus$ symbol marks the atmospheric absorptions.

follow-up discloses the evolution of strong high velocity features in both Si II and Ca II absorption lines. This data set represents a unique opportunity to study the properties of high velocity features and to investigate their origin.

\section{Observations and data set}

SN 2005cf was discovered in the nearby galaxy MCG-01-39003 on a KAIT unfiltered image taken May 2005, 28.36 UT (Puckett et al. 2005). A spectrum taken on May 31.22 UT (Modjaz et al. 2005) showed it to be a type Ia SN about ten days before maximum light. Its host galaxy recession heliocentric velocity is $1937 \mathrm{~km} \mathrm{~s}^{-1}$ (de Vaucouleurs 1991, source NED).

The ESC extensively followed SN 2005cf both photometrically and spectroscopically in the optical and in the near infrared. The photometry data set is discussed in Pastorello et al. (2007). The optical spectroscopy data set presented here covers the time interval from -11.6 days before $B$-band maximum light up to 77.3 days afterwards.

All the data (except those observed with the IFU PMAS) were reduced according to the standard procedure. The two-dimensional images were bias-subtracted and flat-fielded with standard IRAF routines.

Wavelength and flux calibration were applied to the onedimensional optimal extracted spectra (Horne 1986) using calibration observations usually taken with the same instrumental setting and during the same night as the science observations. Atmospheric extinction correction was applied via tabulated extinction coefficients for each telescope used. The PMAS data were reduced using $\mathrm{R}_{3} \mathrm{D}^{2}$, a package for reducing IFS data (Sánchez 2006).

All the spectra were observed at parallactic angle. Synthetic spectrophotometry was computed to adjust the overall accuracy of the flux calibration to photometric data and no substantial flux correction was required. Pastorello et al. (2007) find that the host galaxy reddening was negligible, so no host galaxy reddening correction was performed. According to Schlegel et al. (1998), galactic reddening in the direction of SN $2005 \mathrm{cf}$ is $E(B-V)=$ 0.097 , i.e. $A_{B} \sim 0.4$ mag.

\footnotetext{
${ }^{2}$ http://www.caha.es/sanchez/r3d/
} 
The obtained spectral time sequence is shown in Fig. 1 and the details of the data set are in Table 1.

\section{Data analysis}

\subsection{Spectral time sequence}

The ESC spectroscopically followed SN 2005cf for a period of about 3 months starting from 11.6 days before $B$-band maximum light. Figure 1 shows the complete time sequence. The first spectrum at day -11.6 shows deep absorptions due to Si II, $\mathrm{Mg}$ II, Fe III, and Ca II typical of type Ia SNe. From a first inspection of this spectrum, Si II and Ca II lines appear to be exceptionally broad. Si II $\lambda 6355$ has a flat bottom that develops to a narrower feature by one week before maximum light. Both the IR triplet and the H\&K components of Ca II show broad absorptions evolving with time toward the appearance of an extra trough on their red side. The morphological evolution of these absorptions will be the subject of the analysis and discussion in the following sections.

A weak S II "W-shaped" line is visible around $5500 \AA$. It strengthens with time and by day +16.4 has completely merged with S II $\lambda 5972$ and with the strong emerging Na I line forming the characteristic wide absorption with long, straight blue edge at around $5700 \AA$. The Fe II-blend absorption feature profile below $5000 \AA$ at day -11.6 is broad and almost featureless. On the red edge of this absorption, two small notches are visible and develop into a deep trough by one week prior to maximum light. From day +25.5 on SN 2005cf enters, the nebular phase and the spectra show the typical Fe II and Co II emission lines.

Besides the exceptionally broad Si II and Ca II absorption features, the spectral evolution of SN 2005cf goes along the line of normal type Ia SNe.

\subsection{Type la SNe comparison of spectra}

The spectral time sequence of SN 2005cf (shown in Fig. 1) highlights the peculiar line profile of some absorption features, which is interesting to compare to those of other normal type Ia SNe with similar $\Delta m_{15}$.

In Fig. 2 the spectrum of SN 2005cf at day -11.6 is compared with those of other type Ia SNe at similar epochs. Early supernova spectroscopy discloses the physical properties of the ejecta's outermost layers where type Ia $\mathrm{SNe}$ show the highest degree of heterogeneity. The spectral absorption features are those of normal type Ia SNe before maximum light. The Ca II H\&K and Si II $\lambda 4130$ absorptions have a similar line profile and location as in the other SNe shown. The Fe III $\lambda 4404$, Mg II $\lambda 4481$, and Si III $\lambda 4580$ form the typical trough near $4300 \AA$. The SN 2005cf and SN 2002bo are very similar in this region, while the other shown spectra have different line profiles. The shallower profile of SN 2003du is probably due to a weaker Mg II component, while SN 1990N and SN 1994D have a stronger Si III line. In the region 4500-5200 $\AA$, SN $2005 \mathrm{cf}$ shows a narrower absorption than the other type Ia SNe with two easily distinguished minima due to Si III $\lambda 5051$ and Fe III $\lambda 5129$. This is due to a shallower Fe II-blend component that in the other objects contributes to making the absorption wider. The S II "W"-shaped absorption and the Si II $\lambda 5972$ are present in all the spectra shown in Fig. 2. The main difference in the five spectra compared are around the $6000 \AA$ and $8000 \AA$ regions. The Si II $\lambda 6355$ of SN 2005 cf appears broad and deep with a flat bottom similar to that of SN 1990N. In SN 1994D at 10 days prior

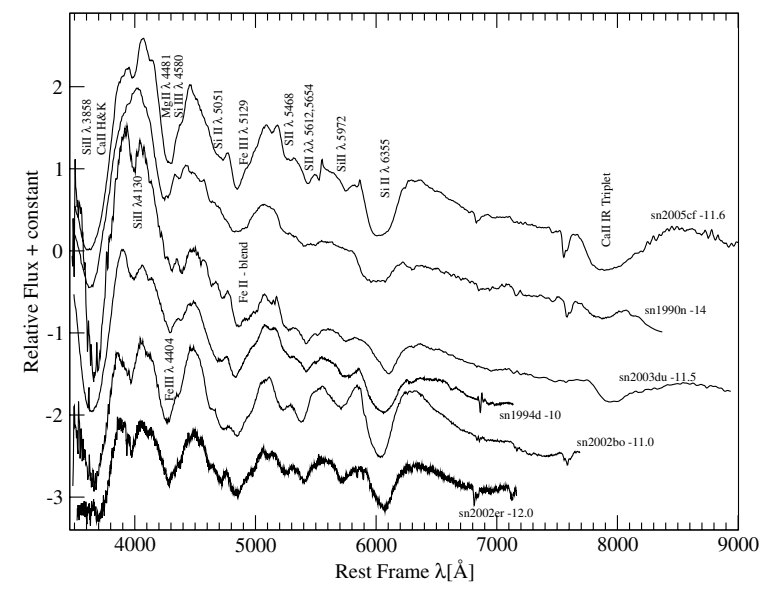

Fig. 2. SN 2005cf in comparison with other $\mathrm{SNe}$ at day -11.6 (SN 1990N, Leibundgut et al. 1991; SN 1994D, Patat et al. 1996; SN 2003du, Stanishev et al. 2007; SN 2002bo, Benetti et al. 2004; SN 2002er, Kotak et al. 2005).

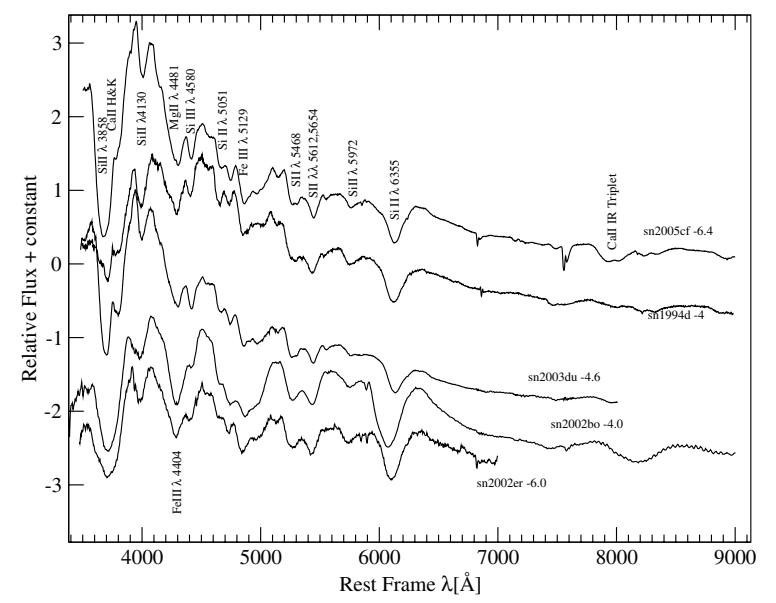

Fig. 3. $\mathrm{SN} 2005 \mathrm{cf}$ in comparison with other $\mathrm{SNe}$ at day -6.4 , (SN 1994D, Patat et al. 1996; SN 2003du, Stanishev et al. 2007; SN 2002bo, Benetti et al. 2004; SN 2002er, Kotak et al. 2005).

to maximum light, this feature is as wide but not as deep and doesn't have a flat bottom. The line profile in SN 2003du is different. The minimum of the absorption feature appears to lie at longer wavelengths than in SN 2005cf, but the trough spans approximately the same wavelength range. The blue edge appears less steep. Meanwhile SN 2002er and SN 2002bo have similar line profiles but different depths. The Ca II triplet region is not covered in all the spectra shown in Fig. 2. In SN 2005cf this feature appears to be at shorter wavelengths and is deeper and wider than in SN 2003du.

In Fig. 3 the comparison between the spectrum of SN 2005cf at about -6.4 days and that of other $\mathrm{SNe}$ is shown. The spectrum of SN 2005cf has evolved while maintaining some of the characteristics already shown in the earlier epochs. Most of the absorption features developed along the lines of normal type Ia SNe, and now the spectrum looks very similar to that of SN 2003du at about 5 days before maximum light. The main differences stand out on Si II 16355, Ca II H\&K and IR Triplet. While Ca II H\&K on SN 2005cf has just a hint of a second minimum on the red side of the absorption, SN 1994D and SN 2003du show a well-developed one. Instead, SN 2002bo and SN 2002er present a broad feature with a single minimum trough. The absorption feature around $6100 \AA$ (i.e. Si II 26355) in SN 2005cf appears 


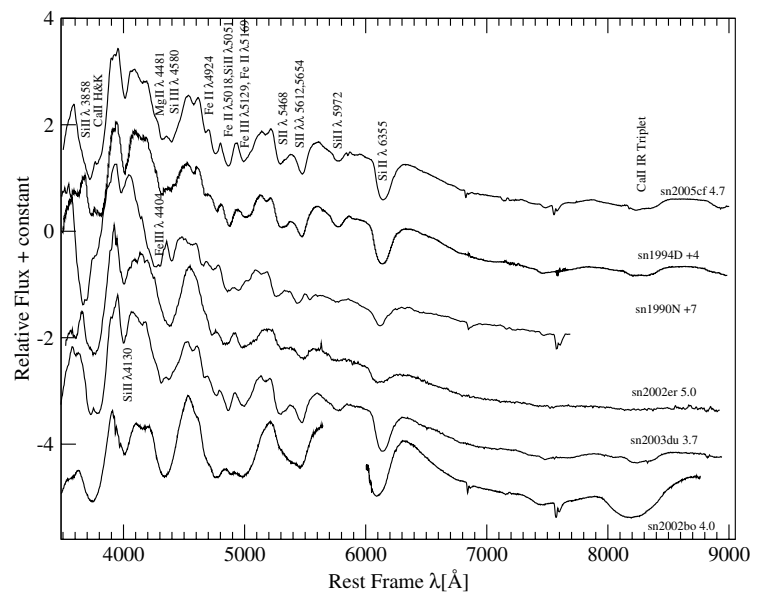

Fig. 4. SN 2005cf in comparison with other $\mathrm{SNe}$ at day +4.7, (SN 1990N, Leibundgut et al. 1991; SN 1994D, Patat et al. 1996; SN 2003du, Stanishev et al. 2007; SN 2002bo, Benetti et al. 2004; SN 2002er, Kotak et al. 2005).

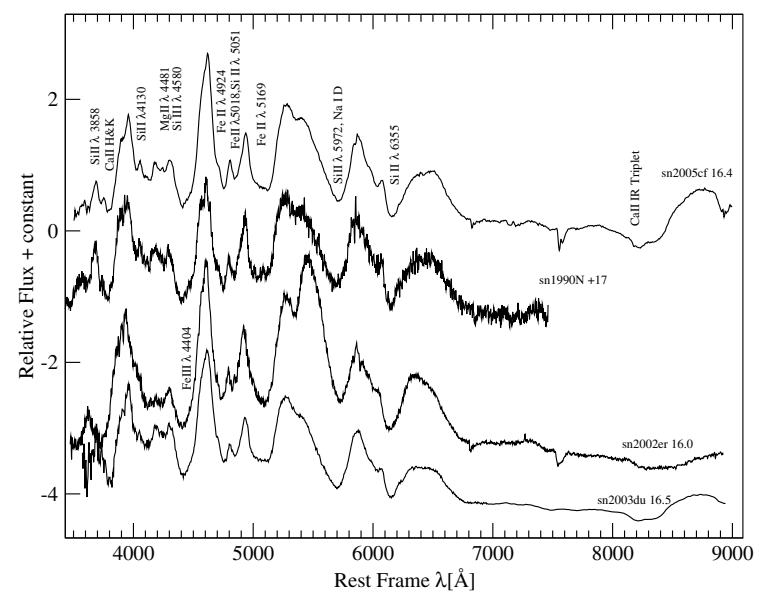

Fig. 5. SN 2005cf in comparison with other $\mathrm{SNe}$ at day +16.4 , (SN 1990N, Leibundgut et al. 1991; SN 2003du, Stanishev et al. 2007; SN 2002er, Kotak et al. 2005).

to be less deep and wide than in SN 2002bo and SN 2002er, but similar to SN 2003du. On its blue edge, a small notch remains visible as a residual of the blue component. The Ca II IR triplet in SN 2005cf now shows a weaker blue feature, which developed a double-minimum line profile and a red component that is gaining strength. In comparison with the other type Ia SNe, both components appear deeper than in SN 1994D. Instead SN 2002bo has a stronger blue but a weaker red component. The spectra of the other objects shown in Fig. 3 do not cover this wavelength region.

About 5 days after maximum light, the spectra of SN 2005cf (shown in Fig. 4), SN 2003du and SN 1994D appear to be very similar, while SN 2002bo shows wider absorption features all across the wavelength span and a deep Ca II IR triplet at highvelocity (i.e. on the blue side of the feature). SN 2003du is the closest match to SN 2005cf in this region. The absorptions on the blue edge of Si II $\lambda 6355$ and in Ca II IR triplet have faded away in SN 2005cf.

At +16.4 days after maximum light type Ia $\mathrm{SNe}$ generally show a smaller degree of spectral diversity. In Fig. 5 the compared spectra appear all to be similar. Stronger Fe II lines have appeared in the wavelength region around $5000 \AA$, and the $\mathrm{Na}$ I D line now dominates the over "W"-shaped S II absorption.

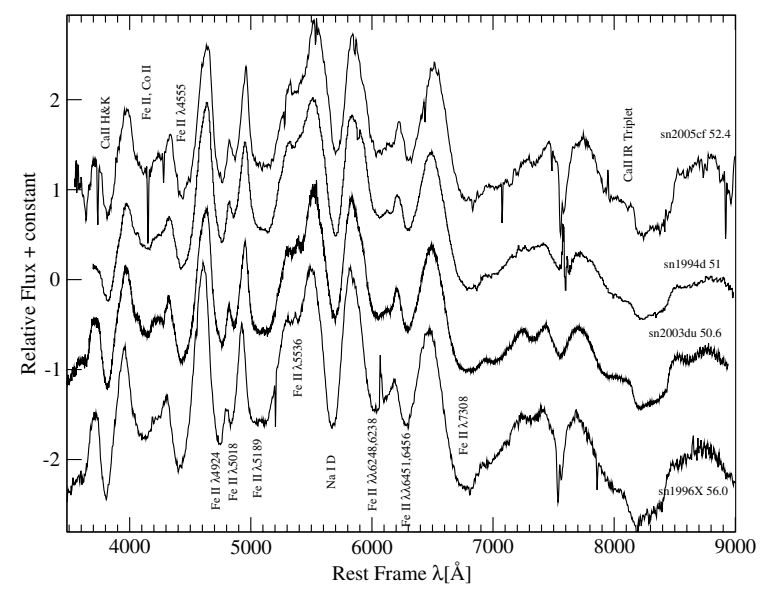

Fig. 6. $\mathrm{SN} 2005 \mathrm{cf}$ in comparison with other $\mathrm{SNe}$ at day +52.4 , (SN 1994D, Patat et al. 1996; SN 2003du, Stanishev et al. 2007; SN 1996X, Salvo et al. 2001).

Some degree of diversity is still visible in the intensity of the Ca II IR triplet. SN 2005cf has a deep absorption with a pronounced emission component, while SN 2002er shows a much weaker feature. SN 2002er also shows a unique emission peak at around $5500 \AA$.

In the early nebular phase, at about +52 days, the spectra compared in Fig. 6 look very homogeneous. All the spectra show similar line profiles and intensities in all their emission features.

\subsection{Ejecta velocity structure}

The analysis carried out in the previous sections highlights that SN 2005cf is overall a normal type Ia SNe with two peculiarities. During the first week of our observations, Si II $\lambda 6355$ appears to be broader than in most of the other type Ia SNe. Ca II absorption lines are also broader and shifted toward bluer wavelength. These characteristics are usually interpreted as signs of the presence of high-velocity material.

\subsubsection{A high-velocity silicon feature?}

In Fig. 7 the time scan of approximately the first three weeks of spectral evolution of SN 2005cf is shown, highlighting the Doppler blueshift of Si II 26355 . At day -11.6 , the line profile is wide with a flat bottom ranging between 12000 and $18000 \mathrm{~km} \mathrm{~s}^{-1}$. As the spectra evolve, the blue side of the absorption loses strength and by day -3.6 the feature shows a single minimum at about $11000 \mathrm{~km} \mathrm{~s}^{-1}$. The line profile is still affected by the blue component until around maximum light when Si II $\lambda 6355$ appears as a single-minimum line at $10000 \mathrm{~km} \mathrm{~s}^{-1}$ with a steep blue edge.

One interpretation of the peculiar time-evolution of the line profile of Si II $\lambda 6355$ can be given by modeling the spectra with the use of the parametrized code for supernova synthetic spectroscopy SYNOW (Fisher 2000). SYNOW is a direct analysis code that generates spectra based on a simple, conceptual model of an SN appropriate during the first few weeks to months after explosion. This model consists of a blackbody-emitting, sharply defined photosphere surrounded by an extended line-forming, pure scattering, homologously expanding atmosphere. For each ion introduced, the Sobolev optical depth as a function of velocity for a "reference line" (usually a strong optical line) is specified. Optical depths in other lines of the ion are set assuming 


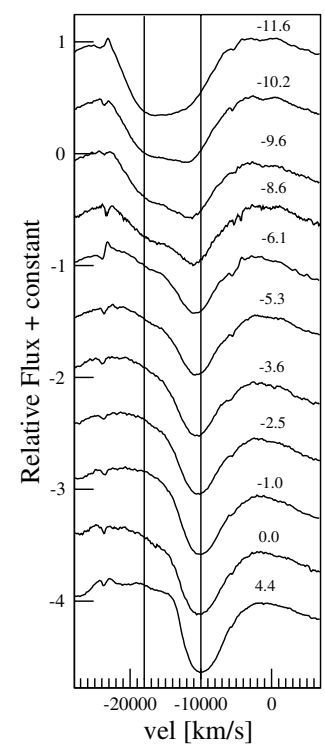

Fig. 7. The first three weeks of evolution in velocity space of Si II $\lambda 6355$ for SN $2005 \mathrm{cf}$. Vertical lines indicate 10000 and $18000 \mathrm{~km} \mathrm{~s}^{-1}$.

Boltzmann excitation of the levels at temperature $T_{\text {exc }}$. The parameters $v_{\text {phot }}$ and $T_{\mathrm{bb}}$ set the velocity and blackbody continuum temperature of the photosphere, respectively. For each ion, the optical depth $\tau$ at the minimum ejection velocity $v_{\min }$ is specified. Optical depth scales exponentially with velocity according to the $e$-folding velocity parameter $v_{e}$ and is considered to be zero for velocity higher than $v_{\max }$. If $v_{\min }>v_{\text {phot }}$ for an ion, we refer to the ion as "detached".

In our analysis we are interested in constraining the velocity distribution of Si II that appears to be unusual for type Ia SNe. The Si II line visible at about $6150 \AA$ is probably the only SN Ia feature unaffected by line blending. To constrain the Si II velocity distribution within the ejecta of SN 2005cf, it is thus sufficient to simulate the time evolution of only Si II. We then used SYNOW to produce synthetic spectroscopy with only this ion. In Fig. 8 the synthetic spectra with and without highvelocity Si II are compared with the data obtained during the first week. Table 2 reports the parameters used for the simulation. The HVF appears to be detached at about $19500 \mathrm{~km} \mathrm{~s}^{-1}$ with the optical depth decreasing with time. Figure 8 shows that including a detached high-velocity layer to match the HVF in addition to a photospheric velocity layer can accurately reproduce the time evolution of this region's line profile.

We reached this conclusion by varying the key parameters through their plausible ranges. We reasoned as follows. The observed broad line profile of Si II $\lambda 6355$ could be obtained by simultaneously varying the parameters $v_{e}, v_{\min }$, and $v_{\text {phot }}$ for a single or a double component of Si II. Figure 9 shows six of the synthetic spectra we obtained by varying these parameters (and setting all the others as for the best fit, i.e. fit number 1 in Fig. 9) while looking for the best match the SN 2005cf spectrum at -10.2 days.

The only way to cover the Si II whole wavelength span with only one component (i.e. the PVC) is to increase its $e$-folding velocity, $v_{e}$. Fit number 2 in Fig. 9 shows the result of setting $v_{e}=6.3$ for the PVC. The blue steep profile and the flat bottom cannot be accurately reproduced. Introducing a HVC helps in reproducing the steep blue edge and to cover the whole wavelength span. Fits numbers 3 and 4 show the effect of setting the parameter $v_{\min }$ for the HVC, to $v_{\min }=17.5$ and to $v_{\min }=21.5$
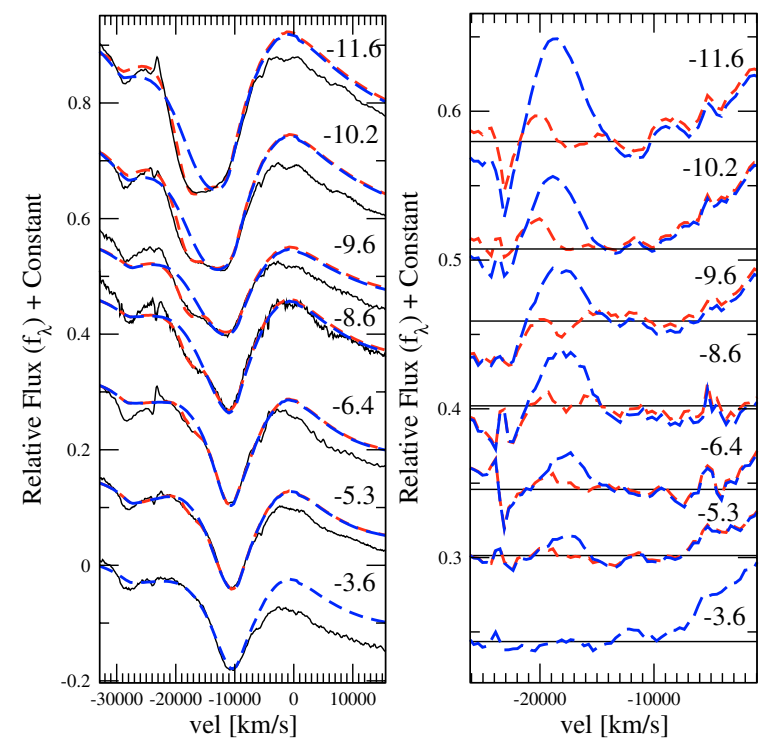

Fig. 8. The evolution in velocity space of Si II $\lambda 6355$ in SN $2005 \mathrm{cf}$ compared with the synthetic models produced with SYNOW (left) and residuals to the observed spectra (right). Long dashed blue lines show SYNOW fits without high velocity Si II. Short dashed red lines show SYNOW fits with high-velocity Si II. See Table 2 for details.

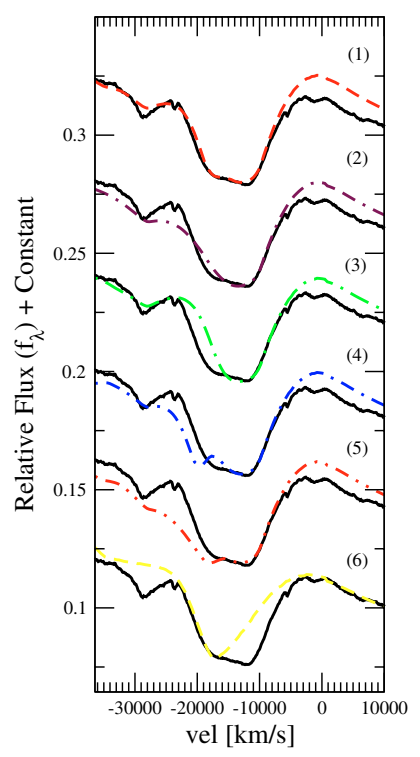

Fig. 9. The velocity space distribution of Si II $\lambda 6355$ in the -10.2 days spectrum of SN 2005cf compared with a variety of synthetic spectra produced with SYNOW. The different synthetic spectra reproduce the effect of varying the key parameters through their plausible ranges. (1) Best fit, including both the HVC and PVC. (2) Fit including only the PVC with $v_{e}=6.3$. (3) Parameters as in the best fit but with the $v_{\min }=17.5$ for the HVC. (4) Parameters as in the best fit but with the $v_{\min }=21.5$ for the HVC. (5) Parameters as in the best fit but with the $v_{=} e=6.3$ for the HVC. (6) Fit including only the HVC detached at $v_{\text {phot }}=21000 \mathrm{~km} \mathrm{~s}^{-1}$.

respectively. Varying $v_{e}$ on the HVC changes the position of the HVC minimum and the profile of its blue edge (as shown in fit number 5, $v_{e}=6.3$ ). The best fit (fit number 1 in Fig. 9) is the result of fine tuning both $v_{\min }$ and $v_{e}$ for both Si II components (in addition to matching the optical depths $\tau$ ). If PVC is removed and $v_{\text {phot }}$ increased to $21000 \mathrm{~km} \mathrm{~s}^{-1}$, only the blue part of the line profile can be accurately reproduced (fit number 6 ). 

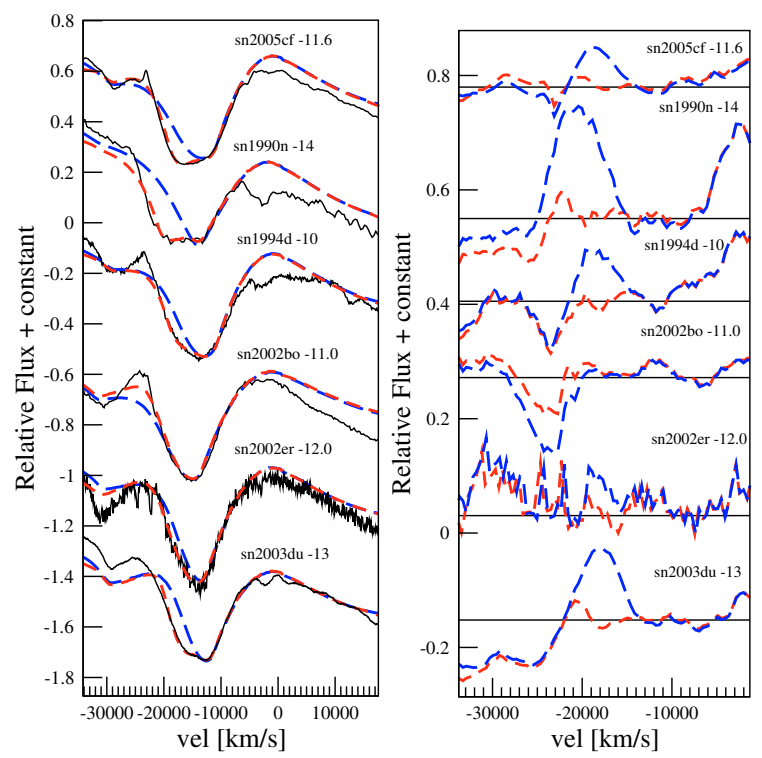

Fig. 10. Comparison of the Si II $\lambda 6355$ line profile of different type Ia $\mathrm{SNe}$ at around day -11.6 in velocity space (left) and residuals to the observed spectra (right). Long dashed blue lines show SYNOW fits without high velocity Si II. Short dashed red lines show SYNOW fits with high-velocity Si II. See Table 3 for details.

The evolution of the relative strength of the two components of Si II in SN 2005cf appears qualitatively similar to the example reported in Fig. 12 of Tanaka et al. (2006). These authors explored the effect of different photosphere-covering factors due to high-velocity material applied to a spectrum at maximum light. The same evolution could be due to the effect of the decreasing optical depth of a high-velocity silicon blob due to the expansion of the supernova ejecta. In order to validate this hypothesis a detailed reconstruction of the three-dimensional ejecta geometry of the SN $2005 \mathrm{cf}$ will be carried out in a forthcoming work (Mazzali et al., in preparation).

\subsubsection{High-velocity $\mathrm{Si}$ II features in other type la $\mathrm{SNe}$}

The presence of an HVF in the Si II $\lambda 6355$ absorption line has been suggested (Mazzali 2001; Mattila et al. 2005; Mazzali et al. 2005b; Stanishev et al. 2007), but it could not yet be securely established for other nearby type Ia SNe. As seen in the case of SN 2005cf, a daily follow up of the pre-maximum spectra can highlight the HVF evolution making the identification possible. Inspecting publicly-available early spectra of type Ia $\mathrm{SNe}$, we found a variety of different Si II $\lambda 6355$ line profiles. We selected the ones that deviated most from the average profile and used SYNOW to simulate their shape.

Figure 10 shows the comparison in velocity space of six type Ia $\mathrm{SNe}$ at day -11.6 in the wavelength region around $6100 \AA$, together with the corresponding SYNOW fits with and without high-velocity Si II. The details of the fits are reported in Table 3 . The intensity and position of the high-velocity component of Si II varies among the different type Ia SNe.

The improvement in the fit achieved when introducing a Si II layer at high velocity is evident in all the cases presented in Fig. 10 with the exception of SN 2002bo, for which the contribution of the high-velocity component is marginal. Therefore, we regard the presence of high-velocity silicon as highly probable in these spectra. However, for a positive identification it would be ideal to closely follow the time evolution of this

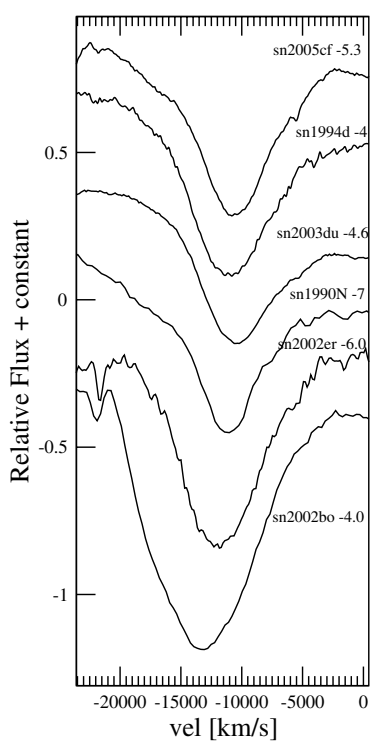

Fig. 11. Comparison of the SiII $\lambda 6355$ line profile of different type Ia SNe at day around -5.3 .

feature, which requires daily spectroscopic observations within the first ten days after the supernova explosion. As it is visible in Fig. 11, by one week before maximum light the optical depth of Si II in the high-velocity layer has dropped to a level that does not have an evident impact on the line profile of Si II $\lambda 6355$. The degree of homogeneity in this line has greatly increased by day around -5 . However, SN 2002bo still has a different line profile due to its overall (i.e. in all its spectral features) exceptionally broad absorption features, and SN 1990N still preserves a hint of the HVF.

\subsubsection{High-velocity Ca II features}

As highlighted by Mazzali et al. (2005a), the presence of high velocity features in the Ca II IR triplet is common in many type Ia SNe during the pre maximum light phase. In all the cases explored in Mazzali et al. (2005a), Ca II IR triplet HVF loses strength with time, and by around maximum light the absorption at $8000 \AA$ is dominated by the photospheric component.

We could expect to see similar behavior in the $H \& \mathrm{~K}$ absorption feature. However, this wavelength region is affected by strong line blending (mainly heavy elements and Si II), so it is more complicated to be certain of the presence of HVFs by synthetic spectroscopy. In the case of SN 2005cf a simple morphological comparison shows similarities between the two absorption features. Figures $12 \mathrm{a}$ and $\mathrm{b}$ show the time scan of Ca II H\&K and IR Triplet, between day -11.6 and day +4.7 respectively, in Doppler blue-shift velocity space.

In the first spectrum, both features show high-velocity minima at about $24000 \mathrm{~km} \mathrm{~s}^{-1}$. The subsequent evolution of the two wavelength regions is similar. A second minimum gradually develops at lower velocity (at about $10000 \mathrm{~km} \mathrm{~s}^{-1}$ ) and the corresponding absorption features strengthen with time. By maximum light, the IR triplet photospheric velocity feature reaches the same depths as the high-velocity one. The same is true one week later for $\mathrm{Ca}$ II $\mathrm{H} \& \mathrm{~K}$. 

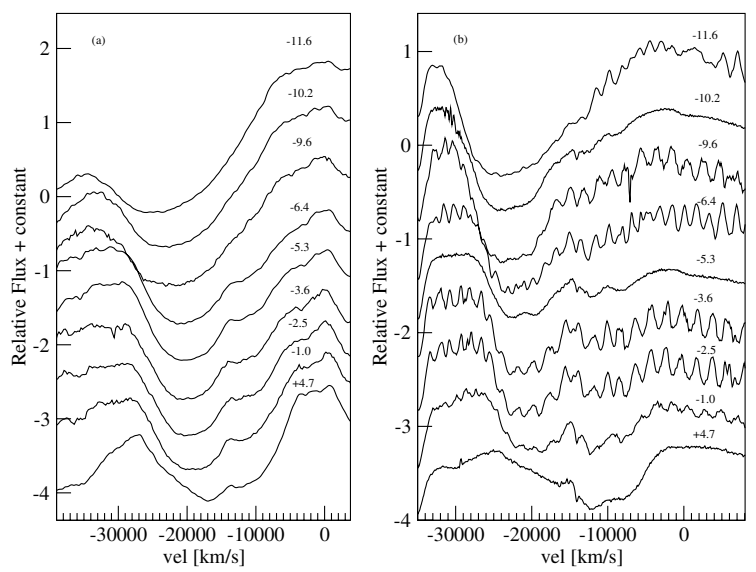

Fig. 12. a) SN 2005cf time scan in velocity space for Ca II H\&K. b) SN 2005cf time scan in velocity space for Ca II IR triplet.

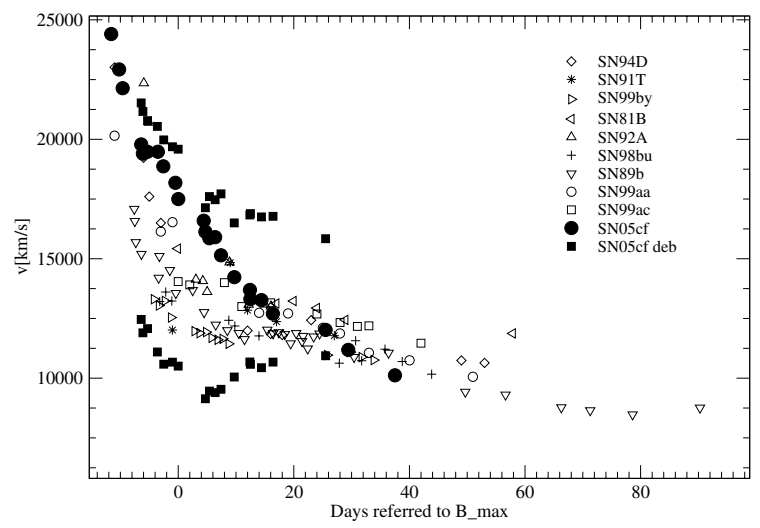

Fig. 13. Expansion velocities as measured from the minima of $\mathrm{Ca}$ II H\&K of SN 2005cf compared with the values of other SNe taken from Wells et al. (1994), Garnavich et al. (2004), Kirshner et al. (1993), Patat et al. (1996), Jha et al. (1999), Garavini et al. (2005), and references therein. Values for SN 2005cf are marked as filled circles when fitting the whole feature with a single Gaussian model. Filled squared symbols mark the results obtained when a deblending with a double Gaussian model was performed. Measured values for the single Gaussian model are reported in Table 4.

\subsubsection{Si II and Ca II velocity time evolution}

The time evolution of Si II $\lambda 6355$ and Ca II H\&K is interesting to look at in more detail, to investigate the expansion velocity as a function of the time at which these absorption features are formed.

Figure 13 shows the Ca II $H \& K$ expansion velocity as a function of time with respect to the $B$-band maximum light for SN 2005cf and other type Ia SNe. The plotted values (filled circles) are the result of fitting a Gaussian profile to the whole Ca II $\mathrm{H} \& \mathrm{~K}$ absorption line profile. Between 6 days before and about 10 days after maximum, the values measured for SN 2005cf appear higher than the average due to the persistent presence of the Ca II H\&K HVF in SN 2005cf, which offsets the measured velocities toward higher values. At a given phase the relative strength of the high and low velocity Ca II H\&K features for different type Ia SNe can differ, so can its time evolution. In the case of SN 2005cf, the Ca II H\&K HVF dominates the Ca II H\&K region for an unusually long period of time, up to one week after maximum light, and remains visible up to approximately three weeks after maximum. Therefore comparing the velocity measured by fitting the whole $\mathrm{Ca}$ II $\mathrm{H} \& \mathrm{~K}$ line profile can be difficult,

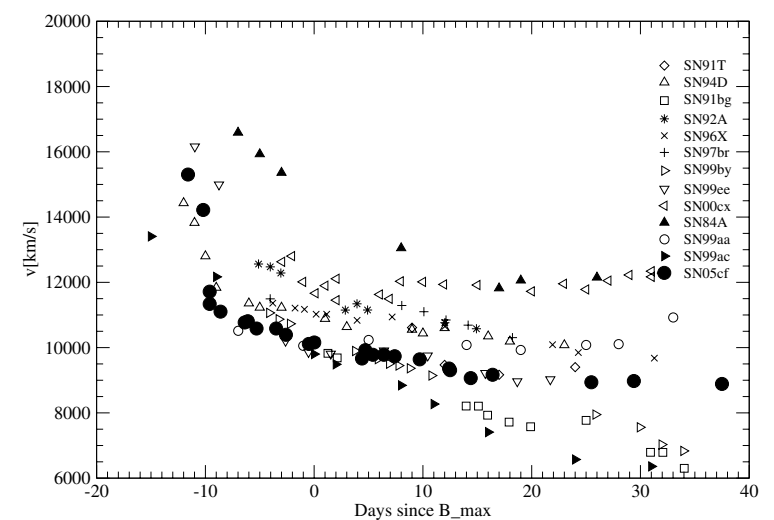

Fig. 14. Expansion velocities as measured from the minima of Si II $\lambda 6355$ of SN $2005 \mathrm{cf}$ compared with the values of other SNe taken from Li et al. (1999), Li et al. (2001), Garnavich et al. (2004), Salvo et al. (2001), Garavini et al. (2005), and references therein. Values for $\mathrm{SN} 2005 \mathrm{cf}$ are marked as filled circles. Measured values are reported in Table 4.

and only qualitative conclusions should be derived from such a comparison.

Figure 13 shows also the results of fitting the Ca II H\&K profile with a double Gaussian model (filled squares) identifying when possible the two visible minima. These values are however, only indicative of the position of the two Ca II H\&K components. Only an accurate synthetic spectroscopy study of the Ca II H\&K absorption feature, including all the ions contributing to the region, could constrain the velocity distribution of the high and low velocity components of Ca II H\&K.

Figure 14 shows the Si II expansion velocity as a function of time since the $B$-band maximum light for SN $2005 \mathrm{cf}$ and other type Ia SNe. The time evolution of the Si II velocity appears to be similar to that of SN 1999ee, showing values on the lower edge of normal type Ia SNe.

\subsection{Possible interpretation of high-velocity features}

Garavini et al. (2004) showed that the presence of HVFs of Ca II, $\mathrm{C}$ II, and Fe II can be reproduced via an artificial, detached line strength in the framework of SYNOW. In the present work we have produced evidence that, in SN 2005cf as well as in other type Ia SNe the same is true for Si II. The physical interpretation of such high-velocity absorption features in terms of the supernova progenitor system evolution and explosion mechanism and dynamics is still under discussion.

The additional line strength in the external layer of the ejecta can be interpreted as either abundance or density enhancements. However, Mazzali et al. (2005b) showed that it is not possible to reproduce strong HVFs by just letting $\mathrm{Si}$ and $\mathrm{Ca}$ dominate the outermost layers of the supernova ejecta. Density enhancements must also be involved. Three-dimensional explosion models show that it is possible to create local density enhancements in the form of blobs of incomplete silicon-burning material at high-velocity (Röpke et al. 2006). This scenario is supported by the strong polarization of some of the HVFs observed in some type Ia SNe, see for example Wang et al. (2003). Alternatively Quimby et al. (2006) and Gerardy et al. (2004) propose that high velocity density enhancement could be the result of an interaction of the ejecta with a circumstellar shell of solar composition.

To disentangle these different scenarios or to understand their interplay is beyond the scope of this analysis. Eventually, only daily spectropolarimetric follow-up observations from the 
Table 5. Measured values of $\mathrm{RJD}_{B}^{\max }, M_{B}^{\max }, \Delta m_{15}, \mathcal{R}(\mathrm{Si}$ II $), v_{10}(\mathrm{Si}$ II $), \dot{v}$ for SN $2005 \mathrm{cf}$. Errors are indicated in parenthesis.

\begin{tabular}{ll}
\hline \hline $\mathrm{RJD}_{B}^{\max }$ & $53534.0^{*}$ \\
$M_{B}^{\max }$ & $-19.39(0.33)^{*}$ \\
$\Delta m_{15}$ & $1.12(0.03)^{*}$ \\
$\mathcal{R}(\mathrm{Si} \mathrm{II})$ & $0.27(0.03)$ \\
$v_{10}(\mathrm{Si}$ II $)$ & $9939(300)$ \\
$\dot{v}$ & $35(5)$ \\
\hline
\end{tabular}

* As measured in Pastorello et al. (2007).

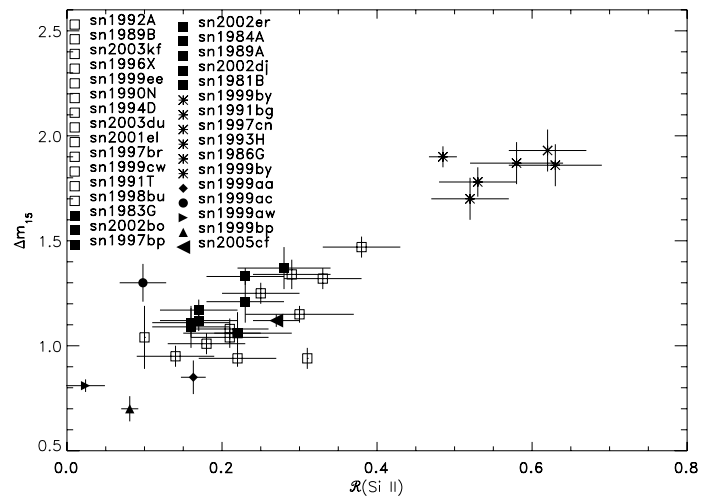

Fig. 15. Light curve decline rate $\Delta m_{15}$ versus $\mathcal{R}$ (Si II). The symbols are chosen accordingly with in Benetti et al. (2005). Open squares mark $\mathrm{SNe}$ belonging to the LVG group, filled squares mark the HVG group, and starred symbols mark the FAINT group. Other filled symbols mark the objects presented in Garavini et al. (2005) and SN 2005cf.

very first days of the explosion will provide the necessary data set to disclose the origin of HVFs.

\subsection{Supernova parameter space}

Type Ia supernovae are currently believed to be a multiparameter class of objects. Several parameters have been proposed with the goal of exploring type Ia SNe diversity. However, the exact number of parameters needed to describe the observational evidence is not yet known. In the following we make use of the parametrization proposed by Benetti et al. (2005) to quantitatively compare SN 2005cf with other observed type Ia SNe.

The parameters used for such comparison are: $\mathcal{R}(\mathrm{Si}$ II) (Nugent et al. 1995), $v_{10}$ (Si II) (Branch \& van den Bergh 1993), $\dot{v}$ (Benetti et al. 2005), and $\Delta m_{15}$ (Phillips 1993). The measured values for SN 2005cf are reported in Table 5. The symbols used in the following figures are chosen to match those in Benetti et al. (2005) to maintain the same distinction among the three clusters found in their analysis. According to this parameterization type Ia $\mathrm{SNe}$ can be divided into three groups (see Benetti et al. 2005, for details): FAINT (low luminosity and high $\Delta m_{15}$ ), HVG (high-velocity gradient $\dot{v}$ ), and LVG (low velocity gradient $\dot{v}$ ).

In Fig. 15 the value of $\Delta m_{15}$ versus $\mathcal{R}(\mathrm{Si}$ II) is shown in comparison to other type Ia SNe. According to these parameters SN 2005cf could be identified as belonging to both the HVG or LVG groups. The comparison (shown in Fig. 16) between the values of the light curve decline rate and the Si II velocity at 10 days past maximum light (i.e. $v_{10}$ (Si II)) positions SN $2005 \mathrm{cf}$ at the lower edge of the LVG group showing a lower velocity value. Also in the plane $\Delta m_{15}$ versus $\dot{v}$ (Fig. 17), SN 2005cf belongs to LVG group showing a low-velocity gradient.

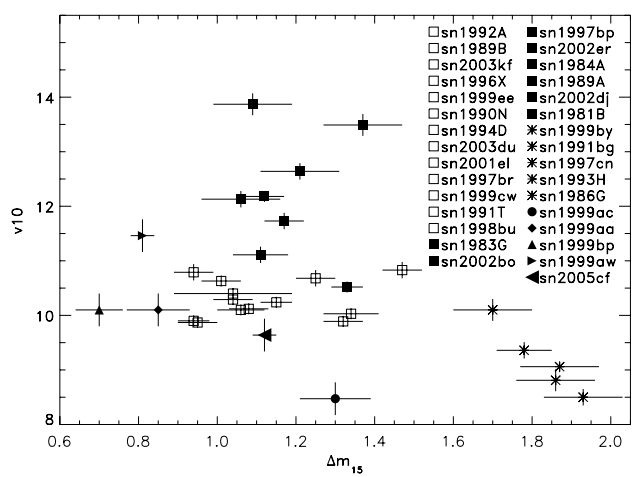

Fig. 16. Plot of $\Delta m_{15}$ versus $v_{10}$ (Si II). Symbols as in Fig. 15.

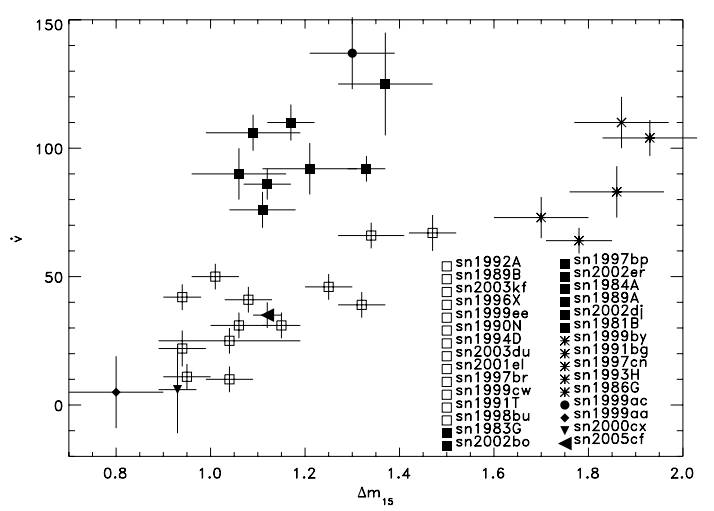

Fig. 17. Plot of $\Delta m_{15}$ versus $\dot{v}$. Symbols as in Fig. 15 .

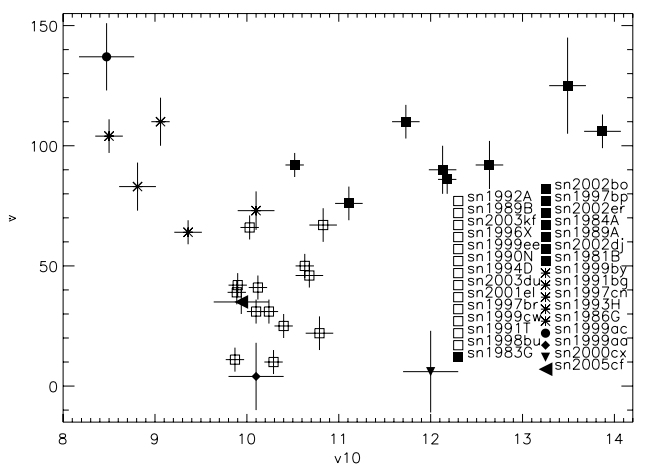

Fig. 18. Plot of $\dot{v}$ versus $v_{10}(\mathrm{Si}$ II). Symbols as in Fig. 15 .

In the $\dot{v}$ versus $v_{10}$ (Si II) plane (Fig. 18) SN 2005cf seems to belong the LVG group showing a low-velocity gradient value, as well as a low silicon velocity at 10 days past maximum light.

In conclusion, SN 2005 cf is definitively a LVG SN Ia in the classification scheme identified in Benetti et al. (2005). The presence of the HVFs does not seem to affect the classification scheme defined in Benetti et al. (2005) since it is mostly defined through the properties of spectra at or after maximum, when the HVFs are less important.

\section{Summary and conclusions}

We have presented the optical spectroscopy of SN 2005cf. The data collected by the ESC-RTN collaboration range from -11.6 to +77.3 days with respect to $B$-band maximum light. Based on the analysis presented in this paper, SN $2005 \mathrm{cf}$ appears to be a normal type Ia SNe with two interesting peculiarities. 
The early supernova spectra show the presence of highvelocity features of both Ca II IR triplet and H\&K detached around $24000 \mathrm{~km} \mathrm{~s}^{-1}$. The appearance of an HVF in the Ca II IR triplet is common to many type Ia SNe (Mazzali et al. 2005a), but the corresponding visibility of a $\mathrm{Ca}$ II $\mathrm{H} \& \mathrm{~K}$ component at high-velocity is not equally widespread.

The second rare characteristic is the clear presence of a detached layer or blob of Si II at about $19500 \mathrm{~km} \mathrm{~s}^{-1}$. We establish the occurrence of the corresponding high-velocity features by means of SYNOW synthetic spectra. Moreover, we also securely identify the presence of such feature in SN 1990N, SN 1994D, SN 2002er, and SN 2003du. This shows that the presence of high-velocity $\mathrm{Si}$ II features is not uncommon in early type Ia $\mathrm{SNe}$ spectra. However, daily early time spectroscopic follow up must be acquired to observe its time evolution.

We have carried out a quantitative comparison between SN 2005cf and other type Ia SNe based on the parameterization presented by Benetti et al. (2005). SN 2005cf appears to belong to the LVG group, showing a slow expansion velocity at 10 days after maximum light, as well as a low-velocity gradient.

Abundance or density enhancements and the interaction of the expanding ejecta with a circumstellar shell with solar composition have been proposed to interpret the peculiar ejecta structure found in type Ia SNe similar to SN 2005cf. Disentangling these explanations will shed light on type Ia $\mathrm{SNe}$ explosion physics and will be the topic of a forthcoming work.

Acknowledgements. This work has been partially supported by the European Union's Human Potential Program "The Physics of type Ia Supernovae", under contract HPRN-CT-2002-00303. G.G, A.G. and V.S. would also like to thank the Göran Gustafsson Foundation for financial support.

This paper is based on observations collected at the Centro Astronómico Hispano Alemán (Calar Alto, Spain), Siding Spring Observatory (Australia), Telescopio Nazionale Galileo, Nordic Optical Telescope (La Palma, Spain), ESO-NTT Telescope (La Silla, Chile). We thank the support astronomers for performing the observations. We thank Thomas Augusteijn, Eija Laurikainen, Karri Muinonen, and Pasi Hakala for giving up part of their time at the Nordic Optical Telescope (NOT), and Jyri Näränen, Thomas Augusteijn, Heiki Salo, Panu Muhli, Tapio Pursimo, Kalle Torstensson, and Danka Parafcz for performing the observations. We are also grateful to P. Sackett for help in observing SN 2005cf from Siding Spring.

This research made use of the NASA/IPAC Extragalactic Database (NED), which is operated by the Jet Propulsion Laboratory, California Institute of Technology, under contract with the National Aeronautics and Space Administration. We also made use of the Lyon-Meudon Extragalactic Database (LEDA), supplied by the LEDA team at the Centre de Recherche Astronomique de Lyon, Observatoire de Lyon.

\section{References}

Astier, P., Guy, J., Regnault, N., et al. 2006, A\&A, 447, 31

Benetti, S., Meikle, P., Stehle, M., et al. 2004, MNRAS, 348, 261

Benetti, S., Cappellaro, E., Mazzali, P. A., et al. 2005, ApJ, 623, 1011

Branch, D., \& van den Bergh, S. 1993, AJ, 105, 2231

Branch, D., Baron, E., Hall, N., Melakayil, M., \& Parrent, J. 2005, PASP, 117, 545

Branch, D., Dang, L. C., Hall, N., et al. 2006, PASP, 118, 560

de Vaucouleurs, G. 1991, Science, 254, 1667

Fisher, A. K. 2000, Ph.D. Thesis

Garavini, G., Folatelli, G., Goobar, A., et al. 2004, AJ, 128, 387

Garavini, G., Aldering, G., Amadon, A., et al. 2005, AJ, 130, 2278

Garnavich, P. M., Kirshner, R. P., Challis, P., et al. 1998, ApJ, 493, L53

Garnavich, P. M., Bonanos, A. Z., Krisciunas, K., et al. 2004, ApJ, 613, 1120

Gerardy, C. L., Höflich, P., Fesen, R. A., et al. 2004, ApJ, 607, 391

Horne, K. 1986, PASP, 98, 609

Jha, S., Garnavich, P. M., Kirshner, R. P., et al. 1999, ApJS, 125, 73

Jha, S., Branch, D., Chornock, R., et al. 2006, AJ, 132, 189

Kirshner, R. P., Jeffery, D. J., Leibundgut, B., et al. 1993, ApJ, 415, 589

Knop, R. A., Aldering, G., Amanullah, R., et al. 2003, ApJ, 598, 102

Kotak, R., Meikle, W. P. S., Pignata, G., et al. 2005, A\&A, 436, 1021

Leibundgut, B., Kirshner, R. P., Filippenko, A. V., et al. 1991, ApJ, 371, L23

Li, W. D., Qiu, Y. L., Qiao, Q. Y., et al. 1999, AJ, 117, 2709

Li, W., Filippenko, A. V., Gates, E., et al. 2001, PASP, 113, 1178

Mattila, S., Lundqvist, P., Sollerman, J., et al. 2005, A\&A, 443, 649

Mazzali, P. A. 2001, MNRAS, 321, 341

Mazzali, P. A., Benetti, S., Altavilla, G., et al. 2005a, ApJ, 623, L37

Mazzali, P. A., Benetti, S., Stehle, M., et al. 2005b, MNRAS, 357, 200

Modjaz, M., Kirshner, R., Challis, P., \& Berlind, P. 2005, IAU Circ., 8534, 3

Nugent, P., Phillips, M., Baron, E., Branch, D., \& Hauschildt, P. 1995, ApJ, 455, L147

Pastorello, A., et al. 2007, MNRS, accepted

Patat, F., Benetti, S., Cappellaro, E., et al. 1996, MNRAS, 278, 111

Perlmutter, S., Aldering, G., della Valle, M., et al. 1998, Nature, 391, 51

Perlmutter, S., Aldering, G., Goldhaber, G., et al. 1999, ApJ, 517, 565

Phillips, M. M. 1993, ApJ, 413, L105

Puckett, T., Langoussis, A., Chen, Y.-T., et al. 2005, IAU Circ., 8534, 1

Quimby, R., Höflich, P., Kannappan, S. J., et al. 2006, ApJ, 636, 400

Riess, A. G., Filippenko, A. V., Challis, P., et al. 1998, AJ, 116, 1009

Riess, A. G., Strolger, L.-G., Tonry, J., et al. 2004, ApJ, 607, 665

Röpke, F. K., Gieseler, M., Reinecke, M., Travaglio, C., \& Hillebrandt, W. 2006, A\&A, 453, 203

Salvo, M. E., Cappellaro, E., Mazzali, P. A., et al. 2001, MNRAS, 321, 254

Sánchez, S. F. 2006, Astron. Nachr., 327, 850

Schlegel, D. J., Finkbeiner, D. P., \& Davis, M. 1998, ApJ, 500, 525

Schmidt, B. P., Suntzeff, N. B., Phillips, M. M., et al. 1998, ApJ, 507, 46

Stanishev, et al. 2007, to be published

Tanaka, M., Mazzali, P. A., Maeda, K., \& Nomoto, K. 2006, ArXiv Astrophysics e-prints

Tonry, J. L., Schmidt, B. P., Barris, B., et al. 2003, ApJ, 594, 1

Wang, L., Baade, D., Höflich, P., et al. 2003, ApJ, 591, 1110

Wells, L. A., Phillips, M. M., Suntzeff, B., et al. 1994, AJ, 108, 2233 
G. Garavini et al.: SN 2005cf, Online Material p 1

\section{Online Material}


Table 1. Data set specifications.

\begin{tabular}{|c|c|c|c|c|c|c|c|}
\hline $\begin{array}{l}\text { JD } \\
-2400000\end{array}$ & $\begin{array}{r}\text { Epoch } \\
\text { ref. } B_{\max }\end{array}$ & Telescope & Instrument & $\begin{array}{c}\lambda \text { range } \\
[\AA]]\end{array}$ & $\begin{array}{l}\text { Exp. time } \\
\text { [s] }\end{array}$ & Airmass & $\begin{array}{r}\text { Resolution } \\
[\AA]]\end{array}$ \\
\hline 53522.4 & -11.6 & TNG & DOLORES & $3182-9935$ & 1000 & 1.3 & 10 \\
\hline 53523.8 & -10.2 & ESO-NTT & EMMI & $3180-9658$ & 1580 & 1.5 & 6 \\
\hline 53524.4 & -9.6 & CA-3.5 & PMAS & $3587-8950$ & 1380 & 1.6 & 7 \\
\hline 53524.4 & -9.6 & NOT & ALFOSC & 3357-9933 & 900 & 1.4 & 13 \\
\hline 53525.4 & -8.6 & CA-3.5 & PMAS & $3698-8730$ & 2000 & 1.5 & 6 \\
\hline 53527.6 & -6.4 & TNG & DOLORES & 3282-9935 & 1300 & 1.3 & 11 \\
\hline 53527.9 & -6.1 & SSO-2.3m & DBSB & $3445-6858$ & 300 & 1.5 & 5 \\
\hline 53528.7 & -5.3 & ESO-NTT & EMMI & $3180-9658$ & 600 & 1.3 & 5 \\
\hline 53530.4 & -3.6 & NOT & ALFOSC & 3344-9934 & 900 & 1.3 & 13 \\
\hline 53531.5 & -2.5 & NOT & ALFOSC & 3314-9936 & 900 & 1.3 & 13 \\
\hline 53533.0 & -1.0 & NOT & ALFOSC & 3181-9936 & 900 & 1.3 & 13 \\
\hline 53534.0 & 0.0 & SSO-2.3m & DBSB & 3181-9158 & 600 & 1.1 & 5 \\
\hline 53538.4 & +4.4 & CA-2.2 & CAFOS & 3552-9936 & 1200 & 1.4 & 12 \\
\hline 53538.7 & +4.7 & ESO-NTT & EMMI & $3180-9659$ & 600 & 1.2 & 5 \\
\hline 53539.4 & +5.4 & CA-2.2 & CAFOS & $3552-9936$ & 1800 & 1.4 & 10 \\
\hline 53540.4 & +6.4 & CA-2.2 & CAFOS & 3238-9932 & 1800 & 1.4 & 10 \\
\hline 53541.4 & +7.4 & CA-2.2 & CAFOS & 3314-9932 & 1800 & 1.4 & 10 \\
\hline 53543.7 & +9.7 & ESO-NTT & EMMI & $3215-9658$ & 600 & 1.7 & 4 \\
\hline 53546.4 & +12.4 & CA-2.2 & CAFOS & 3313-9933 & 1800 & 1.5 & 12 \\
\hline 53546.5 & +12.5 & NOT & ALFOSC & 3380-9934 & 1200 & 1.2 & 13 \\
\hline 53558.4 & +14.4 & CA-2.2 & CAFOS & 3454-9933 & 2400 & 1.5 & 11 \\
\hline 53550.4 & +16.4 & CA-2.2 & CAFOS & 3454-9933 & 2400 & 1.4 & 10 \\
\hline 53559.5 & +25.5 & NOT & ALFOSC & 3510-9936 & 1200 & 1.6 & 12 \\
\hline 53563.4 & +29.4 & CA-2.2 & CAFOS & $3430-9935$ & 1800 & 1.6 & 12 \\
\hline 53571.5 & +37.5 & ESO-NTT & EMMI & $3180-9658$ & 1650 & 1.1 & 5 \\
\hline 53581.4 & +47.4 & CA-2.2 & CAFOS & 3587-9932 & 2700 & 1.7 & 10 \\
\hline 53586.4 & +52.4 & CA-2.2 & CAFOS & 3545-9932 & 2700 & 2 & 10 \\
\hline 53595.4 & +61.4 & CA-2.2 & CAFOS & $3897-8709$ & 3600 & 2.2 & 12 \\
\hline 53609.9 & +75.9 & CA-2.2 & CAFOS & 3572-9932 & 2700 & 2.2 & 12 \\
\hline 53611.3 & +77.3 & CA-2.2 & CAFOS & 3439-8731 & 2700 & 2.6 & 13 \\
\hline
\end{tabular}

Table 2. SYNOW parameters for the Si II fits shown in Fig. 8.

\begin{tabular}{l|lllll|llll|l}
\hline \hline & \multicolumn{9}{|c|}{ PVF } & \multicolumn{4}{c|}{ HVF } & \\
\hline day & $v_{\text {phot }}$ & $v_{\max }$ & $\tau$ & $T_{\text {exc }}$ & $v_{e}$ & $v_{\min }$ & $\tau$ & $T_{\text {exc }}$ & $v_{e}$ & $T_{\mathrm{bb}}$ \\
& $10^{3} \mathrm{~km} \mathrm{~s}^{-1}$ & $10^{3} \mathrm{~km} \mathrm{~s}^{-1}$ & & $10^{3} \mathrm{~K}$ & $10^{3} \mathrm{~km} \mathrm{~s}^{-1}$ & $10^{3} \mathrm{~km} \mathrm{~s}^{-1}$ & & $10^{3} \mathrm{~K}$ & $10^{3} \mathrm{~km} \mathrm{~s}^{-1}$ & $10^{3} \mathrm{~K}$ \\
\hline-11.6 & 12 & 19.5 & 5.5 & 10 & 3.8 & 19.5 & 2.5 & 10 & 2 & 11 \\
-10.2 & 11 & 19.5 & 6.2 & 10 & 3.8 & 19.5 & 1.64 & 10 & 2.2 & 11 \\
-9.6 & 10.8 & 19.5 & 5 & 10 & 3.2 & 19.5 & 1.02 & 10 & 2 & 14 \\
-8.6 & 10.8 & 19.3 & 3 & 10 & 3 & 19.3 & 0.5 & 10 & 2 & 9.5 \\
-6.4 & 11.3 & 19.5 & 3 & 10 & 2 & 19.5 & 0.22 & 10 & 1 & 9.7 \\
-5.3 & 10.5 & 19.5 & 5 & 10 & 2 & 19.5 & 0.2 & 10 & 1 & 11 \\
-3.6 & 10.5 & - & 3 & 10 & 2.15 & - & - & - & - & 11 \\
\hline
\end{tabular}

Note. PVF indicates the parametres for the photospheric velocity component. HVF indicates the parameters for the high-velocity component.

Table 3. SYNOW parameters for the Si II fits shown in Fig. 10.

\begin{tabular}{|c|c|c|c|c|c|c|c|c|c|c|c|}
\hline & & & & $\overline{P \text { PVF }}$ & & & & & 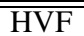 & & \\
\hline SN & Day & $\begin{array}{l}v_{\text {phot }} \\
10^{3} \mathrm{~km} \mathrm{~s}^{-1}\end{array}$ & $\begin{array}{l}v_{\max } \\
10^{3} \mathrm{~km} \mathrm{~s}^{-1}\end{array}$ & $\tau$ & $\begin{array}{l}T_{\text {exc }} \\
10^{3} \mathrm{~K}\end{array}$ & $\begin{array}{l}v_{e} \\
10^{3} \mathrm{~km} \mathrm{~s}^{-1}\end{array}$ & $\begin{array}{l}v_{\min } \\
10^{3} \mathrm{~km} \mathrm{~s}^{-1}\end{array}$ & $\bar{\tau}$ & $\begin{array}{l}T_{\text {exc }} \\
10^{3} \mathrm{~K}\end{array}$ & $\begin{array}{l}v_{e} \\
10^{3} \mathrm{~km} \mathrm{~s}^{-1}\end{array}$ & $\begin{array}{l}T_{\mathrm{bb}} \\
10^{3} \mathrm{~K}\end{array}$ \\
\hline $1990 \mathrm{~N}$ & -14 & 14.6 & 21.9 & 1.15 & 4 & 10 & 21.9 & 0.72 & 10 & 3 & 11.5 \\
\hline 1994D & -11 & 12 & 20 & 7 & 8 & 3 & 20 & 1.2 & 8 & 2 & 12.5 \\
\hline 2002bo & -11 & 15 & 24.5 & 7.5 & 10 & 3 & 24.5 & 0.3 & 10 & 1 & 7.5 \\
\hline 2002er & -12 & 14 & 20 & 7 & 10 & 2.4 & 20 & 1.1 & 10 & 1.8 & 8 \\
\hline $2003 \mathrm{du}$ & -13 & 12.8 & 20 & 10 & 10 & 2 & 20 & 2.1 & 10 & 1 & 16 \\
\hline
\end{tabular}

Note. PVF indicates the parametres for the photospheric velocity component. HVF indicates the parameters for the high-velocity component. 


\section{G. Garavini et al.: SN 2005cf, Online Material p 3}

Table 4. Measurements of the expansion velocity (non-relativist Doppler blushift) inferred from CaII H\&K and Si II $\lambda 6355$ for SN 2005cf.

\begin{tabular}{ccc}
\hline $\begin{array}{c}\text { Epoch } \\
\text { days }\end{array}$ & $\begin{array}{c}\text { Ca II H\&K } \\
{\left[\mathrm{km} \mathrm{s}^{-1}\right]}\end{array}$ & $\begin{array}{c}\text { Si II } \\
{\left[\mathrm{km} \mathrm{s}^{-1}\right]}\end{array}$ \\
\hline-11.6 & 24403 & 15304 \\
-10.2 & 22920 & 14218 \\
-9.6 & - & 11339 \\
-9.6 & 22137 & 11716 \\
-8.6 & - & 11103 \\
-6.4 & 19780 & 10772 \\
-6.1 & 19400 & 10810 \\
-5.3 & 19476 & 10585 \\
-3.5 & 19476 & 10583 \\
-2.6 & 18867 & 10395 \\
-0.5 & 18183 & 10111 \\
-0. & 17499 & 10159 \\
4.4 & 16586 & 9663 \\
4.7 & 16130 & 9923 \\
5.4 & 15856 & 9781 \\
6.4 & 15902 & 9781 \\
7.4 & 15141 & 9734 \\
9.7 & 14229 & 9639 \\
12.4 & 13696 & 9356 \\
12.5 & 13316 & 9309 \\
14.4 & 13267 & 9064 \\
16.4 & 12708 & 9167 \\
25.5 & 12023 & 8936 \\
29.4 & 11187 & 8978 \\
37.5 & 10122 & 8884 \\
\hline & &
\end{tabular}

